\title{
Estresse e estratégias de coping em trabalhadores da saúde de um Centro de Atenção
}

\section{Psicossocial}

\author{
Stress and coping strategies in health workers at a Psychosocial Care Center \\ Estrategias de afrontamiento y estrés en trabajadores de salud de un Centro de Atención \\ Psicosocial
}

Recebido: 09/03/2021 | Revisado: 17/03/2021 | Aceito: 20/03/2021 | Publicado: 28/03/2021

Lucas Barbosa Santos Dias

ORCID: https://orcid.org/0000-0003-1184-691X Universidade do Estado do Rio de Janeiro, Brasil

E-mail: enflucasdias@gmail.com

Elias Barbosa de Oliveira

ORCID: https://orcid.org/0000-0001-5834-7312 Universidade do Estado do Rio de Janeiro, Brasil E-mail: eliasbouerj@gmail.com

Tiago Braga do Espirito Santo ORCID: https://orcid.org/0000-0002-4313-6894 Universidade do Estado do Rio de Janeiro, Brasil E-mail: tbes81@gmail.com

Raphael Lopes Valério

ORCID: https://orcid.org/0000-0002-7406-7983 Universidade do Estado do Rio de Janeiro, Brasil E-mail: raphael_rlv@hotmail.com

Silvia Regina Carvalho de Souza da Silva ORCID: https://orcid.org/0000-0003-2497-9930 Centro de Atenção Psicossocial Rubens Corrêa, Brasil E-mail: silvinhanurse@yahoo.com.br

Anália da Silva Barbosa ORCID: https://orcid.org/0000-0003-4221-9425 Universidade do Estado do Rio de Janeiro, Brasil E-mail: analia.socialrj@gmail.com

\begin{abstract}
Resumo
O presente artigo tem como objetivo verificar as estratégias de coping utilizadas pelos trabalhadores da saúde frente a demanda de acolhimento de usuários em um Centro de Atenção Psicossocial. Trata-se de um estudo transversal descritivo com uma amostra de 14 profissionais de um Centro de Atenção Psicossocial, situado no município do Rio de Janeiro (RJ) em 2019, que responderam questões sociodemográficas e ocupacionais e a Escala de Coping Ocupacional. No tratamento dos dados trabalhou-se com a análise estatística descritiva. Nos resultados é possível observar que a amostra foi composta majoritariamente pelo sexo feminino, solteiros e faixa etária entre 25 e 34 , vínculo empregatício terceirizado e apenas uma fonte de renda. Dentre os fatores e respectivas estratégias de coping utilizadas frequentemente e sempre, identificou-se majoritariamente o controle mediante ações voltadas para a resolução de problemas e suporte social, seguidos do fator manejo de sintomas., principalmente fora do ambiente ocupacional, mediante pratica de exercícios físicos, lazer e mudança de hábitos alimentares. Sobre as estratégias de esquiva, pouco expressivas na amostra, identificou-se que os trabalhadores recorrem a regulação da emoção no intuito de minimizar o estresse ocupacional. Conclui-se que a prevalência das estratégias centradas no controle e no manejo dos sintomas evidenciam estresse ocupacional e riscos para a saúde dos trabalhadores.
\end{abstract}

Palavras-chave: Saúde mental; Saúde do trabalhador; Adaptação psicológica; Estresse ocupacional.

\begin{abstract}
This article aims to verify the coping strategies used by health workers in view of the demand for welcoming users in a psychosocial care center. This is a descriptive cross-sectional study with a sample of 14 professionals from a Psychosocial Care Center located in the city of Rio de Janeiro (RJ) in 2019, who answered sociodemographic and occupational questions and the Occupational Coping Scale. In the treatment of the data, we worked with descriptive statistical analysis. In the results, it is possible to observe that the sample was composed mainly of females, singles and age group between 25 and 34, outsourced employment and only one source of income. Among the factors and respective coping strategies used frequently and always, the control factor was mostly identified through actions aimed at problem solving and social support followed by the symptom management factor, mainly outside the
\end{abstract}


occupational environment through leisure activities, physical exercise and change in eating habits. Regarding avoidance strategies, which are not very significant in the sample, it was identified that workers resort to emotion regulation in order to minimize occupational stress. It is concluded that the prevalence of strategies centered on the control and management of symptoms in the sample shows occupational stress and health risks for workers.

Keywords: Mental health; Occupational health; Adaptation, Psychological; Occupational stress.

\section{Resumen}

Este artículo tiene como objetivo verificar las estrategias de afrontamiento que utilizan los trabajadores de la salud ante la demanda de acogida de usuarios en un Centro de Atención Psicosocial. Se trata de un estudio descriptivo transversal con una muestra de 14 profesionales de un Centro de Atención Psicosocial, ubicado en la ciudad de Rio de Janeiro (RJ) en 2019, quienes respondieron preguntas sociodemográficas, ocupacionales y la Escala de Afrontamiento Ocupacional. En el tratamiento de los datos se trabajó con el análisis estadístico descriptivo. En los resultados se puede observar que la muestra estuvo compuesta principalmente por mujeres, solteros y grupo de edad entre 25 y 34 años, empleo subcontratado y una sola fuente de ingresos. Entre los factores y las respectivas estrategias de afrontamiento utilizadas frecuentemente y siempre, el control se identificó mayoritariamente a través de acciones orientadas a la resolución de problemas y el apoyo social, seguido del factor de manejo de síntomas., Principalmente fuera del ámbito laboral, a través del ejercicio físico., Ocio y cambio en la alimentación. Hábitos. En cuanto a las estrategias de evitación, que no son muy significativas en la muestra, se identificó que los trabajadores recurren a la regulación de las emociones para minimizar el estrés laboral. Se concluye que la prevalencia de estrategias enfocadas al control y manejo de síntomas muestra estrés ocupacional y riesgos para la salud de los trabajadores.

Palabras clave: Salud mental; Salud laboral; Adaptación psicológica; Estrés laboral.

\section{Introdução}

A Reforma Psiquiátrica ocorrida no Brasil ao longo das últimas décadas do século passado e atual (Amarante, 2017), promoveu expressivas mudanças nos saberes, práticas e relações estabelecidas entre profissionais de saúde mental, usuários e familiares. Com a diminuição progressiva do número de leitos e hospitais psiquiátricos, foram introduzidas novas abordagens e espaços de acolhimento dos usuários não mais centradas na lógica manicomial e excludente, porém na oferta de cuidados de base territorial com participação ativa da família.

Dentre os serviços substitutivos do hospital psiquiátrico (Lancetti \& Amarante, 2017) os Centros de Atenção Psicossocial (CAPS) em suas diferentes modalidades de atendimento são pontos de atenção estratégicos da Rede de Atenção Psicossocial (RAPS), de caráter aberto e comunitário constituído por equipe multiprofissional e organizado sob a ótica interdisciplinar. O primeiro CAPS foi criado em 1987, na cidade de São Paulo cuja modalidade de atendimento foi inspirada no movimento italiano de desinstitucionalização para a assistência às pessoas em sofrimento psíquico, incluindo aquelas em situações de crise, com histórico de dependência química ou nos processos de reabilitação psicossocial.

Se por um lado o trabalho realizado no CAPS é visto como gratificante, diferenciado e inovador (Clementino et al, 2018) por sua vez há problemas cuja ausência de resolutividade tem dificultado a consolidação das mudanças propostas no modelo e gerando sofrimento psíquico nos trabalhadores. Esses trabalhadores convivem em seu dia a dia com inúmeras dificuldades e dentre elas: a demanda assistencial crescente, a falta de articulação da rede assistencial intra e intersetorial, a vulnerabilidade social da população atendida e suas precárias condições de vida em geral, a violência no território, o déficit de recursos humanos, materiais e de infraestrutura nos serviços e a terceirização.

Além dos aspectos estruturais e organizacionais dos CAPS (Zanatta, Lucca, Sobral, Stephan \& Bandini, 2019), devese considerar a maior vulnerabilidade dos próprios trabalhadores diante do cuidado de pessoas em sofrimento psíquico e social, cuja exposição contínua pode desencadear estresse e doenças relacionadas ao trabalho, principalmente quando a capacidade adaptativa do indivíduo é ultrapassada e as estratégias de enfrentamento não se mostram eficazes. O estresse ocupacional ocorre nas situações onde há o conflito entre o desejo do trabalhador comprometido com o bem-estar e a saúde dos usuários ao se ver impedido de realizar o trabalho satisfatoriamente.

Estudos sobre a saúde mental com profissionais de várias categorias (Benetti et al, 2015; Melo, Carlotto, Rodriguez \&

Diehl, 2016; Zanata et al, 2019), apontam que o estresse ocupacional é um dos principais riscos ao bem-estar psicossocial do 
indivíduo, com implicações fisiológicas, psicológicas e comportamentais. O desequilíbrio resultante do estresse ocasiona consequências negativas no desempenho profissional, baixa moral, alta rotatividade, absenteísmo e violência laboral. Cabem ao trabalhador e a organização conhecerem e elaborarem mecanismos de enfrentamento saudáveis que minimizem o impacto dos estressores na saúde e na qualidade do atendimento dos usuários. Ratifica-se a relevância das estratégias de coping como preditores da saúde ocupacional, sendo as mesmas determinantes na evolução do estresse e no desenvolvimento de doenças.

Segundo Lazarus e Folmann (1984), o conceito de estratégias de enfrentamento (coping) compreende o conjunto de medidas intencionais, cognitivas e comportamentais adotado pelo indivíduo para se adaptar a diferentes circunstâncias estressantes com o propósito de minimizar sua susceptibilidade e retornar ao seu estado anterior. Tais medidas ao serem adotadas podem ser focalizadas no problema ou na emoção, compreendendo um esforço para regular o estado somático ou emocional e reduzir a sensação física desagradável gerada.

Pelo fato de as pessoas, em sua grande maioria, se encontrarem susceptíveis ao estresse decorrente de estímulos nocivos provenientes do ambiente em que se encontram (Demerouti, 2014), o modo como enfrentam os estressores é relevante no sentido de se minimizar os seus efeitos deletérios para a saúde. $\mathrm{Na}$ área ocupacional os estudiosos ratificam o valor inestimável das características individuais que podem funcionar como atenuadoras dos estressores ou ao contrário, potencializa-los, visto que seus efeitos são resultantes da valoração que a pessoa atribui ao evento.

No intuito de contribuir com os estudos sobre as estratégias de coping no ambiente ocupacional, Latack (1986) desenvolveu a Escala de Coping Ocupacional (ECO), cujas ações e reavaliações cognitivas (de enfrentamento ou de esquiva) realizadas pelo indivíduo frente aos estressores devem estar juntas. Tais estratégias são divididas em três fatores: controle, referente às ações e reavaliações de caráter cognitivo proativo; esquiva, relativos às ações e reavaliações que sugerem fuga ou evitação; manejo de sintomas, que dizem respeito às estratégias utilizadas pelos indivíduos para administrar situações de estresse, incluindo relaxamento ou atividade física.

Objetivo do estudo: verificar as estratégias de coping utilizadas pelos trabalhadores da saúde frente a demanda de acolhimento de usuários em um Centro de Atenção Psicossocial.

\section{Metodologia}

Estudo transversal, descritivo e exploratório. A pesquisa exploratória busca levantar questões ou problemas, com o objetivo de aumentar o conhecimento do pesquisador do ambiente, fato ou fenômeno para estudos futuros (Marconi \& Lakatos, 2017). O projeto foi aprovado pelo Comitê de Ética em Pesquisa (CEP/HUPE) e protocolado no Comitê Nacional de Ética em Pesquisa mediante parecer de número 3.110.646.

O campo foi um CAPS situado no município do Rio de Janeiro, que se encontra capacitado para realizar prioritariamente o atendimento de pacientes com transtornos mentais severos e persistentes em sua área territorial. Esta unidade integra a RAPS do município, responsável pelo atendimento de usuários residentes na Área Programática (AP) 2.2 que inclui os bairros: Praça da Bandeira, Tijuca, Alto da Boa Vista, Maracanã, Vila Isabel, Andaraí, Grajaú e Usina. A equipe do CAPS trabalha em conjunto com onze (11) Clínicas da Família do entorno, possuindo atualmente 317 usuários cadastrados, 57 usuários em processo de recepção, com uma frequência diária que oscila entre 60 a 80 atendimentos.

Em atendimento à Resolução 466/12, após a assinatura do Termo de Consentimento Livre e Esclarecido, de um total de 24 profissionais que atuavam no CAPS, na ocasião em que os dados foram coletados, a amostra foi composta por 14(58,3\%) profissionais. Na elegibilidade dos participantes trabalhou-se com os seguintes critérios: profissionais de formação superior e que participavam da construção do projeto terapêutico singular; vínculo empregatício celetista ou contrato temporário; atuação no serviço há mais de seis meses. Excluídos os profissionais que se encontravam licenciados para tratamento médico, de férias e afastados devido a problemas de outra natureza. 
Informou-se aos participantes que a inclusão no estudo seria voluntária e garantiu-se o direito de se retirarem da pesquisa em qualquer fase, sem que isso trouxesse qualquer dano ou retaliação. Ratificou-se o anonimato e explicou-se que os resultados seriam apresentados em eventos científicos e publicados em revistas científicas. Os dados foram coletados individualmente no período de janeiro a fevereiro de 2019, em local livre de interferências após agendamento e disponibilidade dos profissionais, tendo durado em torno de 20 minutos. Os instrumentos foram preenchidos pelo próprio participante e o responsável pela coleta manteve-se no local para sanar dúvidas, sendo respeitado o distanciamento para evitar desconforto ou interferências.

O estudo incluiu as variáveis: sexo, faixa etária, estado civil, profissão, formação em saúde mental, tipo e número de vínculos empregatícios, carga horária semanal, carga horária total considerando outros vínculos empregatícios e as estratégias de coping segundo a ECO.

A ECO é um instrumento de autopreenchimento traduzido, adaptado e validado no país por Pinheiro, Troccóli e Tamayo (2003), sendo a segunda escala mais utilizada na identificação das estratégias de Coping. O formulário é composto por 29 questões distribuídas em escala do tipo Likert de cinco pontos, em que 1 é assinalado para "nunca faço isso", 2 "raramente faço isso", 3 "às vezes faço isso", 4 "frequentemente faço isso" e 5 "sempre faço isso". Esses itens refletem a forma como os indivíduos lidam com os estressores ocupacionais e compõem os três fatores da ECO da seguinte maneira: fator controle (11 itens de 1 a 11) referentes a ações e reavaliações cognitivo proativo; fator esquiva ( 9 itens de 12 a 20) relativos a ações e reavaliações que sugerem fuga; fator manejo dos sintomas ( 9 itens de 21 a 29).

Com o propósito de verificar as estratégias de coping utilizadas pela amostra, foram entregues aos participantes dois instrumentos, sendo o primeiro composto por dados sociodemográficos e ocupacionais e outro com os itens que compõem a ECO precedida da seguinte questão: "Diante da demanda de atendimento no CAPS e o déficit de recursos humanos para um atendimento singular e personalizado, eu...". Ao término da coleta, os dados foram lançados em uma planilha do Excel e a seguir procedeu-se a análise estatística descritiva (números absolutos e relativos). Tendo em vista o quantitativo de participantes, optou-se por discutir apenas as estratégias utilizadas frequentemente e sempre, após obtenção dos escores dos itens de cada fator a partir da escala Likert.

\section{Resultados}

De acordo com os critérios de inclusão adotados e considerando os 24 profissionais que atuavam no acolhimento, a amostra foi composta por 14(58,3\%) participantes (assistentes sociais, enfermeiros e psicólogos). São do sexo feminino $(85,7 \%)$, solteiros $(57,1 \%)$ e faixa etária entre 25 a 34 anos (57,1\%). Quanto as características ocupacionais identificaram-se psicólogos $(71,4 \%)$, terceirizados $(78,6 \%)$, possuíam apenas um vínculo empregatício $(78,6 \%)$, cumpriam carga horária institucional de até 40 horas $(42,9 \%)$ e acima de 50 horas ao considerar outros vínculos $(21,4 \%)$.

Na Tabela 1 são apresentados os valores relativos as estratégias de coping adotadas pelos trabalhadores frente ao estresse decorrente da demanda de atendimento no CAPS. 
Tabela 1: Valores relativos das estratégias de coping utilizadas pela amostra de acordo com a escala de Likert ( $\mathrm{n}=14$ ). Rio de Janeiro, Brasil, 2018.

\begin{tabular}{cccccc}
\hline \multirow{2}{*}{ Fator/Frequência } & \multicolumn{5}{c}{ Valores \% } \\
\cline { 2 - 6 } & Nunca & Raramente & As vezes & Frequentemente & Sempre \\
\hline Controle & 0,73 & 3,94 & 10,1 & $\mathbf{1 3 , 8}$ & $\mathbf{9 , 6}$ \\
Esquiva & 8,12 & 10,1 & 9,06 & $\mathbf{1 , 9 7}$ & $\mathbf{0 , 7 4}$ \\
Manejo dos sintomas & 5,4 & 5,4 & 9,8 & $\mathbf{6 , 1 5}$ & $\mathbf{4 , 2}$ \\
\hline
\end{tabular}

Fonte: Autores (2021).

Como identificado na Tabela 1, houve prevalência do Fator Controle frequentemente e sempre, tendo em vista as ações empregadas pelos participantes no intuito de minimizarem o estresse ocupacional. Inversamente às estratégias de controle, observa-se que o grupo nunca ou raramente recorre as estratégias de esquiva, o que pode estar ancorado na experiência adquirida no acolhimento e o uso de habilidades empáticas em face do sofrimento dos usuários.

Tabela 2: Frequência das estratégias de controle utilizadas pela amostra de acordo com a ECO (n=14). Rio de Janeiro, Brasil, 2018.

\begin{tabular}{|c|c|c|c|c|c|}
\hline \multirow{2}{*}{ Fator/Frequência } & \multicolumn{5}{|c|}{ Frequência $(\%)$} \\
\hline & 1 & 2 & 3 & 4 & 5 \\
\hline 1. Converso com colegas que também estejam envolvidos no problema. & - & - & 7,1 & 21,4 & 71,4 \\
\hline $\begin{array}{l}\text { 2. Tento ver a situação como uma oportunidade para aprender e desenvolver novas } \\
\text { habilidades. }\end{array}$ & - & - & 28,6 & 42,9 & 28,6 \\
\hline 3. Dou atenção extra ao planejamento. & 7,1 & 7,1 & 42,9 & 21,4 & 14,3 \\
\hline $\begin{array}{l}\text { 4. Penso em mim como alguém que sempre consegue se sair bem em situações } \\
\text { como essa. }\end{array}$ & 7,1 & 21,4 & 57,1 & 14,3 & - \\
\hline 5. Penso na situação como desafio. & 7,1 & - & 21,4 & 42,9 & 28,6 \\
\hline 6. Tento trabalhar mais rápida e eficientemente. & - & 14,3 & 14,3 & 57,1 & 14,3 \\
\hline 7. Decido sobre o que deveria ser feito e comunico às demais pessoas envolvidas. & - & 28,6 & 21,3 & 21,4 & 28,6 \\
\hline 8. Esforço-me para fazer o que eu acho que se espera de mim. & - & 7,1 & 28,6 & 50 & 35,7 \\
\hline $\begin{array}{l}\text { 9. Peço conselho a pessoas que, embora esteja fora da situação, possa me ajudar a } \\
\text { pensar sobre o que fazer. }\end{array}$ & - & 7,1 & 14,3 & 57,1 & 21,4 \\
\hline 10. Tento modificar os fatores que causaram a situação. & - & 21,4 & 50 & 14,3 & 14,3 \\
\hline $\begin{array}{l}\text { 11. Envolvo-me mais ainda nas minhas tarefas, se acho que isso pode ajudar a } \\
\text { resolver a questão. }\end{array}$ & - & 7,1 & 7,1 & 50 & 35,7 \\
\hline
\end{tabular}

Nota: 1. NUNCA 2. RARAMENTE 3. AS VEZES

Fonte: Autores (2021).

\section{FREQUENTEMENTE 5. SEMPRE}

Entre as estratégias de controle (Tabela 2), as ações mais utilizadas foram: converso com colegas que também estejam envolvidos no problema; envolvo-me mais ainda nas minhas tarefas se acho que isso pode ajudar a resolver a questão; peço conselho a pessoas que, embora estejam fora da situação, possam me ajudar a pensar sobre o que fazer; e tento ver a situação como uma oportunidade para aprender e desenvolver novas habilidades. 
Tabela 3: Frequência das estratégias de esquiva utilizadas pela amostra de acordo com a ECO ( $n=14)$. Rio de Janeiro, Brasil, 2018 .

\begin{tabular}{lccccc}
\hline \multicolumn{1}{c}{ Fuga-Esquiva } & \multicolumn{3}{c}{ Frequência (\%) } \\
\cline { 2 - 5 } & $\mathbf{1}$ & $\mathbf{2}$ & $\mathbf{3}$ & $\mathbf{4}$ & $\mathbf{5}$ \\
\hline 1. Evito a situação, se possível. & 35,7 & 28,6 & 28,6 & 7,1 & - \\
2. Digo a mim mesmo que o tempo resolve problemas desta natureza. & 35,7 & 35,7 & 28,6 & - & - \\
3. Tento manter distância da situação. & 42,9 & 28,6 & 21,4 & 7,1 & - \\
4. Procuro lembrar que o trabalho não é tudo na vida. & 14,3 & 7,1 & 35,7 & 14,3 & 14,3 \\
5. Antecipo as consequências negativas, preparando-me assim para o pior. & 7,1 & 21,4 & 42,9 & 21,4 & 7,1 \\
6. Delego minhas tarefas a outras pessoas. & 7,1 & 57,1 & 28,6 & - & - \\
7. Mantenho a maior distância possível das pessoas que causaram a situação. & 35,7 & 28,6 & 7,1 & - & - \\
8. Tento não me preocupar com a situação. & 35,7 & 42,9 & 21,4 & - & - \\
9. Concentro-me em fazer prioritariamente aquilo que gosto. & 21,4 & 42,9 & 35,7 & - & - \\
\hline
\end{tabular}

Nota: 1. NUNCA 2. RARAMENTE 3. AS VEZES 4. FREQUENTEMENTE 5. SEMPRE

Fonte: Autores (2021).

De acordo com a Tabela 3, a utilização das estratégias de esquiva são bem menos frequentes tanto em variedade quanto em quantidade em comparação com as de controle, em que se identificou as seguintes ações: procuro lembrar que o trabalho não é tudo na vida e antecipo as consequências negativas, preparando-me assim para o pior.

Tabela 4: Frequência das estratégias de manejo utilizadas pela amostra de acordo com a ECO (n=14). Rio de Janeiro, Brasil, 2018.

\begin{tabular}{lccccc}
\hline \multicolumn{1}{c}{ Manejo dos sintomas } & \multicolumn{5}{c}{ Frequência (\%) } \\
\cline { 2 - 6 } & $\mathbf{1}$ & $\mathbf{2}$ & $\mathbf{3}$ & $\mathbf{4}$ & $\mathbf{5}$ \\
\hline 1. Pratico mais exercícios físicos. & 28,6 & 14,3 & 14,3 & 35,7 & 7,1 \\
2. Uso algum tipo de técnica de relaxamento. & 21,4 & 21,4 & 50 & 7,1 & - \\
3. Procuro a companhia de outras pessoas. & - & 7,1 & 14,3 & 42,9 & 35,7 \\
4. Mudo os meus hábitos alimentares. & 14,3 & 21,4 & 28,6 & 21,4 & 14,3 \\
5. Procuro me envolver em mais atividades de lazer. & - & 7,1 & 28,6 & 42,9 & 21,4 \\
6. Compro alguma coisa. & 35,7 & - & 50 & - & 14,3 \\
7. Tiro alguns dias para descansar. & 14,3 & 35,7 & 35,7 & 7,1 & 7,1 \\
8. Faço uma viagem. & 14,3 & 28,6 & 28,6 & 14,3 & 7,1 \\
9. Torno-me mais sonhador(a). & 28,6 & 35,7 & 35,7 & 7,1 & 14,3 \\
\hline \multicolumn{1}{c}{ 2. RARAMENTE 3. AS VEZES } & 4. FREQUENTEMENTE & 5. SEMPRE \\
Nota: 1. NUNCA & & & & &
\end{tabular}

Sobre as estratégias de manejo dos sintomas (Tabela 4), verificou-se maior utilização das seguintes ações: procuro a companhia de outras pessoas, procuro me envolver em mais atividades de lazer, prático mais exercícios físicos e mudo meus hábitos alimentares. 


\section{Discussão}

Com a reorientação do modelo assistencial em saúde mental no país (Amarante, 2017), o que se almejava no seu projeto inicial era a desinstitucionalização e a construção de uma rede de suporte social capaz de acolher os usuários e possibilitar novas vivencias com vistas a reabilitação psicossocial e assim prescindir da internação psiquiátrica. Para a sua operacionalização houve necessidade de os profissionais incorporarem os princípios da desinstitucionalização e da reabilitação psicossocial, sendo as equipes que compõem os serviços, como identificado no presente estudo, compostas majoritariamente por psicólogos, assistentes sociais e pessoal de enfermagem (enfermeiros e técnicos de enfermagem). Cabe ressaltar que a equipe mínima, não exclui o médico, porém em termos quantitativos e considerando a proposta de trabalho do CAPS, há um número maior de profissionais de outras áreas da saúde.

Tais dados vão ao encontro de estudo acerca da satisfação e sobrecarga do trabalho em profissionais de um CAPS (Clementino et al, 2018), no qual a amostra foi composta majoritariamente por profissionais não médicos, cuja faixa etária situou-se entre 30 e 39 anos, cumpre carga horaria de 40 horas semanais e possuem vínculo empregatício celetista ou temporário. Entre os fatores estressores, verificou-se o número insuficiente de profissionais, insatisfação com os gestores, os parcos recursos materiais e o acúmulo de funções. Acrescentam a esta problemática os baixos salários, as jornadas exorbitantes e a instabilidade empregatícia decorrente dos contratos de trabalho precários, que inviabilizam projetos e trabalho futuros em decorrência dos términos dos contratos com as organizações sociais.

Segundo Barros e Bernardo (2017) a rotina de trabalho no CAPS é desgastante, devido à grande demanda de envolvimento subjetivo e à precariedade das condições de trabalho que fragilizam o vínculo e dificultam o manejo clínico dos usuários. Consequentemente, há a necessidade de adoção de medidas que visem a mudança da lógica precarizante do trabalho em saúde mental, uma vez que a insatisfação no trabalho repercute especialmente na saúde física, mental e social dos profissionais.

Nos CAPS de acordo com Athayde e Hennington (2012), os procedimentos técnicos e padronizados não são tão frequentes, pois o que se almeja de verdade é a reflexão constante sobre os modos de se trabalhar, evitando-se a todo custo a repetição de práticas desumanas e normatizadoras. Nesse contexto predominam regras éticas sobre as de caráter técnico, tais como o trabalho no território com a participação efetiva de usuários, familiares e comunidade. Este trabalho ético, entretanto, não exime o desgaste devido à alta demanda de atendimento aliada à precarização do trabalho (Alves, Dourado \& Côrtes, 2013).

Sobre as estratégias de coping adotadas pelos profissionais do CAPS em face do estresse decorrente da demanda por atendimento, evidenciou-se maior concordância da amostra de afirmativas em relação as ações voltadas para o fator Controle. Os trabalhadores recorrem a ajuda do grupo no intuito de resolver problemas, envolvem-se ainda mais nas tarefas, pedem conselhos, veem o trabalho como uma oportunidade de aprender e desenvolver novas habilidades, pensam na situação como desafio, tentam trabalhar mais rápida e eficientemente e se esforçam para fazer o que acham que se espera do grupo.

Pesquisa sobre o estresse e as estratégias de coping com uma amostra de 193 trabalhadores da saúde em 11 CAPS (Zanata et al, 2019), no qual os autores utilizaram o Inventário de Estratégias de Coping de Folkmann e Lazarus (1984), evidenciou-se que as estratégias mais utilizadas pelos profissionais foram "resolução de problemas" (34,2\%), "suporte social" (26,8\%) e fuga-esquiva" $(19,4 \%)$. As ações de esquiva têm como propósito a regulação das emoções de modo a prevenir o confronto e/ou o afastamento físico do trabalho. Em outro estudo com enfermeiros de um hospital pediátrico hematológico, (Umann, Silva, Benetti \& Azevedo Guido, 2013) o fator Controle foi o mais utilizado no intuito de minimizar o estresse em função do déficit de recursos humanos e materiais. As ações mais empregadas foram: "me esforço para fazer o que eu acho que se espera de mim", "converso com os colegas que também estejam envolvidos no problema" e "tento modificar os fatores que causaram a situação". 
A escolha de determinada estratégia de coping ou ações voltadas para o enfrentamento do estresse ocupacional (Benetti et al, 2015) mantem relação com as características pessoais, cargo ou função desempenhada, tipo de atividade realizada, assim como aquelas relacionadas as experiências e conhecimentos acumulados ao longo da trajetória profissional. Tais fatores em seu conjunto, de acordo com Melo et al, (2016), contribuem para que o indivíduo realize a avaliação primária e secundaria do estressor no intuito de escolher determinada ação e fazer a reavaliação. Portanto, as experiências acumuladas são relevantes na escolha da estratégia de coping, visto que os indivíduos desenvolvem habilidades de enfrentamento a partir de circunstâncias vivenciadas.

Tendo em vista que no presente estudo, a maioria dos participantes afirmou utilizar ações centradas no controle, há de se atentar para o fato de que o trabalho desenvolvido junto aos usuários do CAPS caracteriza-se por alta carga psíquica, tendo em vista o déficit de pessoal e a necessidade de acolhimento do sofrimento psíquico dos usuários e familiares. Ao estabelecer a relação entre as ações de controle e manejo dos sintomas, observou-se a tentativa de minimizar o estresse fora do ambiente de trabalho mediante realização de atividades como: suporte da rede de apoio social, lazer, prática de exercícios físicos e a mudança dos hábitos alimentares. Apenas 1 (um) participante afirmou recorrer frequentemente a técnicas de relaxamento.

Em relação ao fator esquiva, identificou-se que uma parcela de trabalhadores, ao contrário de fugir das situações e/ou eventos estressores, recorreu a regulação das próprias emoções e/ou sentimentos sempre e frequentemente ao optarem pelas afirmativas: "o trabalho não é tudo na vida" e "antecipam as consequências negativas, preparando-se assim para o pior". Cabe a reflexão sobre a eficácia das estratégias de controle e manejo dos sintomas que ao serem adotadas minimizam a fuga ou esquiva frente aos estressores ocupacionais.

\section{Conclusão}

A amostra do estudo foi composta majoritariamente por psicólogos, sexo feminino, solteiros e faixa etária entre 25 e 34, vínculo empregatício terceirizado e apenas uma fonte de renda. Dentre os fatores e respectivas estratégias de coping usadas frequentemente e sempre, identificou-se majoritariamente o fator controle mediante ações voltadas para a resolução de problemas e suporte social. Verificou-se também o manejo de sintomas, principalmente fora do ambiente ocupacional mediante atividades de lazer, prática de exercícios físicos e mudança de hábitos alimentares. Quanto as estratégias de esquiva, pouco presente na amostra, os trabalhadores recorrem a modulação da emoção no intuito de minimizar o seu impacto na saúde mental e física.

Entende-se que a organização do trabalho tem grande influência na saúde mental dos trabalhadores, podendo propiciar tanto o desgaste quanto o prazer no trabalho. Deve-se, portanto, compreender as possibilidades de se intervir nas situações geradoras de sofrimento que ao longo do tempo podem levar ao adoecimento e até mesmo ao abandono dos postos de trabalho. O estudo traz a reflexão sobre a importância das estratégias de coping utilizadas pelos profissionais, no intuito de mitigarem o sofrimento no trabalho e ao mesmo tempo manterem-se nos postos de trabalho diante da demanda de atendimento por parte dos usuários e familiares.

Os resultados podem servir de referência para gestores e profissionais com vistas a elaboração de programas voltados para a prevenção do estresse ocupacional e investimento em condições dignas de trabalho ao considerar o impacto das ações na qualidade do atendimento ofertado aos usuários. Ratifica-se que pelo fato de os trabalhadores do CAPS recorrerem as estratégias de coping no intuito de minimizar os efeitos do estresse sobre o organismo e manter a qualidade do atendimento, cabe a instituição investir em ações preventivas no que diz respeito a ampliação do quadro de pessoal, considerando que a maioria dos profissionais são terceirizados.

Como limitações do estudo, deve-se considerar a opção pelo método transversal que retrata uma situação momentânea sem estabelecer o nexo causal entre exposição e desfecho. O número reduzido de participantes em função da própria 
organização dos CAPS no país, dificulta a generalização dos achados para outros contextos. Há necessidade de ampliação de estudos com um maior número de participantes envolvendo outros CAPS, considerando a importância do treinamento dos profissionais acerca das estratégias de coping na minimização do estresse psicossocial.

\section{Referências}

Amarante, P. (2017). Saúde mental e atenção psicossocial. (4a ed.). SciELO-Editora FIOCRUZ.

Alves, H. M. D. C., Dourado, L. B. R., \& Côrtes, V. D. N. Q. (2013). A influência dos vínculos organizacionais na consolidação dos Centros de Atenção Psicossociais. Ciência \& Saúde Coletiva, 18, 2965-2975.

Athayde, V., \& Hennington, É. A. (2012). A saúde mental dos profissionais de um Centro de Atenção Psicossocial. Physis: Revista de Saúde Coletiva, 22, 983-1001.

Avelino, D. C., de Castro Silva, P. M., Costa, L. D. F. P., de Azevedo, E. B., Saraiva, A. M., \& Filha, M. D. O. F. (2014). Trabalho de enfermagem no centro de atenção psicossocial: estresse e estratégias de coping. Revista de Enfermagem da UFSM, 4(4), 718-726.

Barros, A. C. F., \& Bernardo, M. H. (2017). A lógica neoliberal na saúde pública e suas repercussões para a saúde mental de trabalhadores de CAPS. Revista de Psicologia da Unesp, 16(1), 60-74.

Benetti, E. R. R., Stumm, E. M. F., Weiller, T. H., de Melo Batista, K., Lopes, L. F. D., \& de Azevedo Guido, L. (2015). Estratégias de Coping e características de trabalhadores de enfermagem de hospital privado. Revista da Rede de Enfermagem do Nordeste, 16(1), 3-10.

Clementino, F. D. S., Miranda, F. A. N. D., Martiniano, C. S., Marcolino, E. D. C., Pessoa Júnior, J. M., \& Fernandes, N. M. S. (2018). Avaliação da satisfação e sobrecarga de trabalho dos trabalhadores dos Centros de Atenção Psicossocial. Rev. pesqui. cuid. fundam. (Online), 10(1), 153-159.

Demerouti, E. (2014). Individual strategies to prevent burnout. In Burnout at Work (pp. 40-63). Psychology Press.

Kleinubing, R. E., Goulart, C. T., da Silva, R. M., Umann, J., \& de Azevedo Guido, L. (2013). Estresse e coping em enfermeiros de terapia intensiva adulto e cardiológica. Revista de Enfermagem da UFSM, 3(2), 335-344.

Lancetti, A., \& Amarante, P. (2017). Saúde mental e saúde coletiva. In Tratado de saúde coletiva. (2a ed.), HUCITEC, FIOCRUZ (pp. 628-633).

Latack, J. C. (1986). Coping with job stress: Measures and future directions for scale development. Journal of Applied Psychology, 71 (3), 377.

Lazarus, R. S., \& Folkman, S. (1984). Stress, appraisal, and coping. Springer publishing company.

Marconi, M. D. A., \& Lakatos, E. M. (2017). Fundamentos da metodologia científica. (8a ed.): Editora Atlas SA.

Melo, L. P., Carlotto, M. S., Rodriguez, S. Y. S., \& Diehl, L. (2016). Estratégias de enfrentamento (coping) em trabalhadores: revisão sistemática da literatura nacional. Arquivos Brasileiros de Psicologia, 3(68), 125-144.

Pinheiro, F. A., Tróccoli, B. T., \& Tamayo, M. R. (2003). Mensuração de coping no ambiente ocupacional. Psicologia: teoria e pesquisa, 19(2), 153-158.

Umann, J., da Silva, R. M., Benetti, E. R. R., \& de Azevedo Guido, L. (2013). Estresse e coping entre enfermeiros de unidade hemato-oncológica. Revista da Rede de Enfermagem do Nordeste, 14(4), 783-790.

Umann, J., de Azevedo Guido, L., \& da Silva, R. M. (2014). Estresse, coping e presenteísmo em enfermeiros que assistem pacientes críticos e potencialmente críticos. Revista da Escola de Enfermagem da USP, 48(5), 891-898.

Zanatta, A. B., de Lucca, S. R., Sobral, R. C., Stephan, C., \& Bandini, M. (2019). Stress and coping among workers at psychosocial care centers in the interior of the state of Sao Paulo. Revista Brasileira de Medicina do Trabalho, 17(1), 83. 